\title{
Co action of CFTR and AQP1 increases permeability of peritoneal epithelial cells on estrogen-induced ovarian hyper stimulation syndrome
}

Pei-Yin Jin ${ }^{1,3+}$, Yong-Chao Lu ${ }^{2 \dagger}$, Ling $\mathrm{Li}^{1^{*}}$ and Qin-Fu Han ${ }^{3}$

\begin{abstract}
Background: Ovarian hyper stimulation syndrome (OHSS) is an iatrogenic complication associated with fertility drugs. It is characterized by increased vascular permeability and substantial fluid shift with accumulation in the body cavity. The pathogenesis of OHSS remains obscure, and no definitive treatments are currently available.

Results: Using western blot and short-circuit current (Isc) techniques, we investigate the potential coactions of analysis in cystic fibrosis transmembrane conductance regulator (CFTR) and aquaporin 1 (AQP1) on the hyper permeability of body cavity peritoneal epithelial cells in the pathogenesis of OHSS. The rats develop OHSS symptoms, with the up regulation of both CFTR and AQP1 expression and enhanced CFTR channel activity in peritoneal epithelial cells, can also be mimicked by administration of estrogen, alone in ovariectomized rats. Administration of progesterone suppresses CFTR activity, OHSS symptoms as well as CFTR and AQP1 expression. Besides, AQP1 inhibitor, $\mathrm{HgCl}_{2}$, can suppress CFTR channel activity. Therefore, antisera against CFTR or AQP1 to OHSS animals may result in alleviation of the symptom.

Conclusion: This study confirms the coactions of CFTR and AQP1 play a critical role in the development and progression of increased peritoneal epithelial permeability in severe OHSS. These findings may provide grounds for ameliorating assisted reproduction treatment strategy to reduce the risk of OHSS in in vitro fertilization (IVF).
\end{abstract}

Keywords: CFTR, AQP1, Estrogen, Ovarian hyper stimulation syndrome

\section{Background}

Ovarian hyper stimulation syndrome (OHSS) is a severe complication of ovulation induction in in vitro fertilization (IVF) treatment. The incidence of OHSS occurs in about $0.1-4 \%$ of ovulation induction treatments [1], is increasing around the world through the expansion of infertility treatments. OHSS is characterized by massive cystic enlargement of the ovaries associated with third space fluid shift. This can result in the formation of ascites and pleural effusion in severe conditions due to increased peritoneal epithelial permeability [2]. Severe OHSS can be accompanied by

\footnotetext{
* Correspondence: LiLing63035@sina.com

${ }^{\dagger}$ Equal contributors

'Department of Cardiovascular Medicine, The First Affiliated Hospital of

Zhengzhou University, Zhengzhou, Henan 450052, China

Full list of author information is available at the end of the article
}

hypercoagulability, thromboembolic phenomena, adult respiratory distress syndrome, and even death. Although this syndrome has been identified for a long time, the exact mechanism of OHSS induction is not clear. An increased epithelial permeability is the initial change leading to OHSS [3]. Many cytokines and vasoactive and angiogenic factors, such as vascular endothelial growth factor (VEGF), have been implicated as major mediators of the pathogenesis of capillary leakage and endothelial damage in OHSS [4-7].

Fluid effluxing across epithelial cells is followed by ion movements. Ion movements play a crucial role in the movement of water across epithelial cells, as water fluctuation depends on the opening and closing of aquaporins (AQPs) actively and inactively transported as it follows ion movements according to osmotic gradients

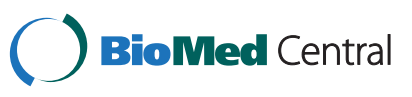

(c) 2012 Jin et al.; licensee BioMed Central Ltd. This is an Open Access article distributed under the terms of the Creative Commons Attribution License (http://creativecommons.org/licenses/by/2.0), which permits unrestricted use, distribution, and reproduction in any medium, provided the original work is properly cited. 
[8]. Rapid passage of fluid into luminal spaces, as seen in OHSS, may be a consequence of abnormal ion transport across the epithelial cells. Several pathological conditions, such as cholera-induced diarrhea, in which there is massive fluid efflux of ion and water across epithelial membranes, are mediated by altered expression and function of transepithelial ion channels, particularly cystic fibrosis transmembrane conductance regulator (CFTR) [9]. Cystic fibrosis is manifested by mutations in CFTR [10], the most common lethal genetic disease in Caucasians, with a hallmark defect in epithelial electrolyte and fluid transport throughout the body [11]. A previous study only focused on the reproductive system effect of CFTR on OHSS, but did not investigate CFTR in the peritoneal epithelial cells [12]. Meanwhile, in the process of fluid effusion, water efflux involves a water (AQP1) pathway by which ion and other osmotic gradients drive water movement [8]. So the coactions of ion channels and AQP1 such as CFTR [12] are important in the process of fluid effusion.

CFTR is expressed in most epithelial cells including the airways and gastrointestinal and reproductive tracts $[13,14]$. Its expression is known to be regulated by ovarian hormones $[15,16]$, upregulated by estrogen, and down regulated by progesterone [17]. Cyclic changes in CFTR expression and its channel activity have been correlated with cyclic changes in uterine fluid volume $[18,19]$. Meanwhile, the water channel AQP1 is expressed strongly throughout microvascular endothelia except the central nervous system, such as in the kidneys, lungs, skins, secretory glands, skeletal muscles, pleura and peritoneum $[20,21]$. AQP1 is involved in fluid transport [20,21], and facilitates water movement from capillaries into the peritoneal cavity [22]. Its expression is also regulated by ovarian hormones [23,24]. It has also been well established that estrogen levels are highly elevated during ovarian hyper stimulation, with excessively high levels observed in patients with OHSS [25,26]. We, therefore, hypothesized that increased estrogen levels during ovarian hyper stimulation may lead to upregulated CFTR and AQP1 expression and channel activity, resulting in the elevated epithelial secretory activity. The most prominent feature of OHSS is marked with ascites or even pleural effusion almost always associated with the administration of human chorionic gonadotropin (hCG) [2,27]. So we chose peritoneal epithelial cell
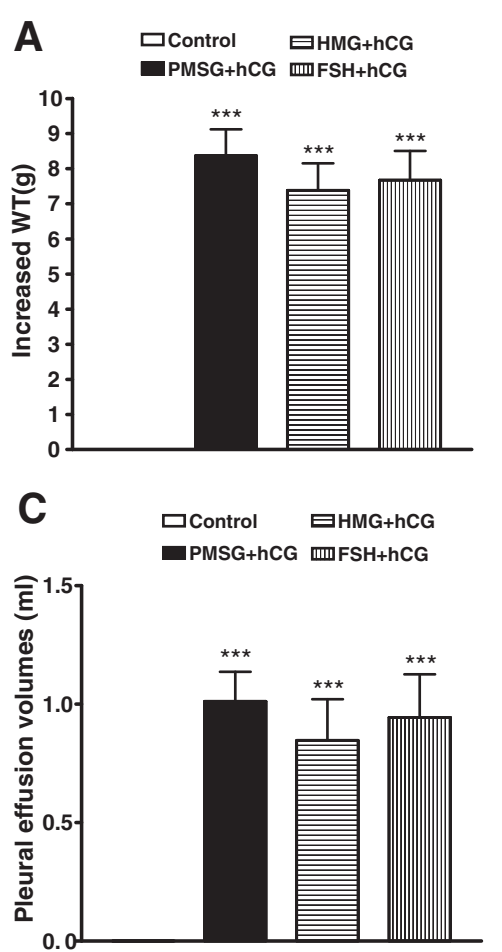

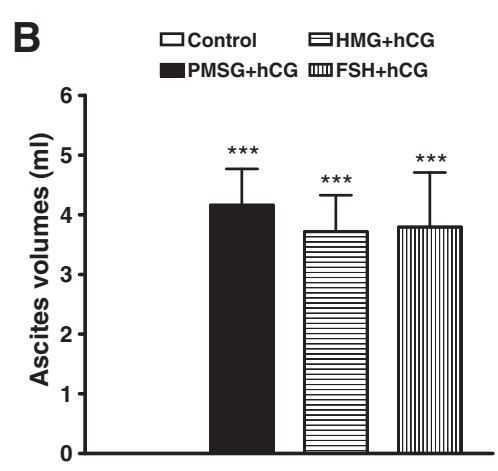

D

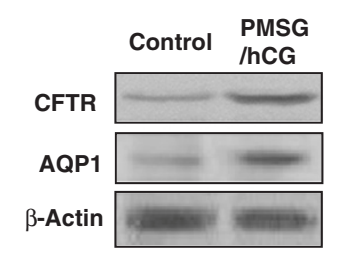

Figure 1 Induction of OHSS rat model and upregulation of CFTR and AQP1 expression in peritoneal epithelial cells. Hyperstimulated rats given different combinations of gonadotropins showed significantly enhanced WT increase, ascites volumes and pleural effusion volumes compared with the controls ( $\mathbf{A}, \mathbf{B}$ and $\mathbf{C} ; \mathrm{n}=5$ ) with corresponding enhancement of CFTR and AQP1 protein expression by western blot in PMSG- and hCG-induced OHSS rat peritoneal epithelial cells. The Pixel Value for CFTR: Control 265488, PMSG/hCG 765729; The Pixel Value for AQP1: Control 150834, PMSG/hCG 348630; The Pixel Value for $\beta$-Actin: Control 980058, PMSG/hCG 971052 (D). ***P<0.001 (compared with the control). 
model for the study of coactions of CFTR and AQP1 in OHSS development.

\section{Results}

Up regulation of CFTR and AQP1 expression in peritoneal epithelial cells

Combinations of gonadotropins including HMG, FSH, or PMSG in conjunction with hCG were used to trigger OHSS. In our study, rat hyper stimulation was defined by the observation of either fluid accumulation in the peritoneal cavity and thorax, or increased body weight (WT) in contrast to the saline-treated control rats (Figure 1 A-C). Body weight was measured, and ascites was collected by performing a lower midline incision and completely draining the intra-abdominal fluid into a flask. After ascites was collected, the diaphragm was cut off by scissors and pleural effusion volume was also collected. The effusion volumes were then measured with a 1 -ml tuberculin syringe respectively. Most prominently, the increase of ascites volume and pleural effusion volume in the hyper stimulated rats was significantly different from that in the controls (Figure $1 \mathrm{~B}$ and $\mathrm{C}$ ). This was associated with elevated CFTR and AQP1 expression in peritoneal epithelial cells (Figure $1 \mathrm{D}$ ).

\section{Estrogen upregulated CFTR and AQP1 expression and induced OHSS in rats}

Estrogen levels were measured in our PMSG/hCGinduced OHSS rats and those control rats at both estrous and diestrous stages of the estrous cycle. More than 5-fold increase in estrogen levels was found in the OHSS rats compared with that in the control (Figure 2 A). We also found that hyper stimulation did not produce OHSS symptom in ovariectomized rats. However, treatment of ovariectomized rats with estrogen $(10 \mu \mathrm{g} / \mathrm{kg} \cdot \mathrm{d})$, but not progesterone $(25 \mathrm{mg} / \mathrm{d})$ for $5 \mathrm{~d}$, induced WT increase compared with ovariectomized control $(P<0.0001$; Figure 2 B) and increased CFTR and AQP1 expression in peritoneal epithelial cells (Figure $2 \mathrm{C}$ ) similar to the OHSS rat model. Furthermore, progesterone suppressed the action of estrogen on OHSS induction and CFTR and AQP1 expression up regulation (Figure $2 \mathrm{~B}$ and $\mathrm{C}$ ). These results suggest that the ovarian sex hormone, estrogen,
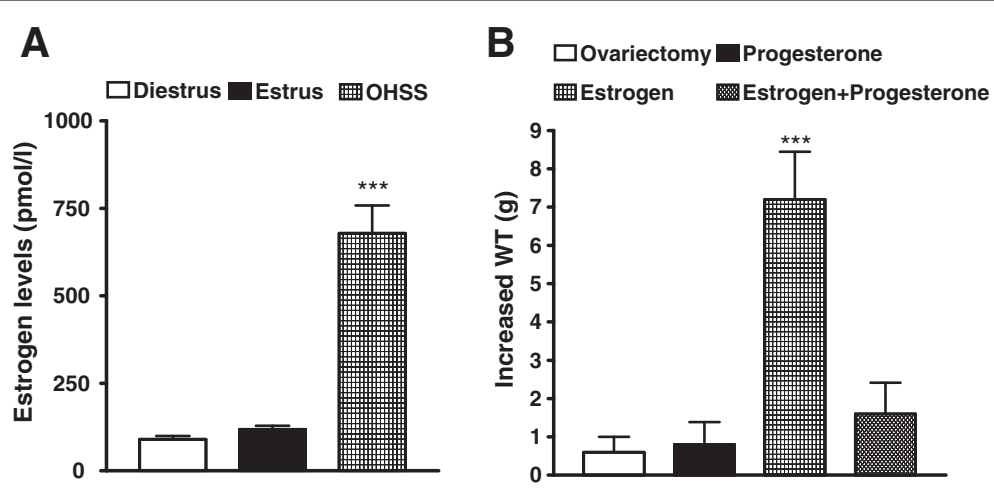

C

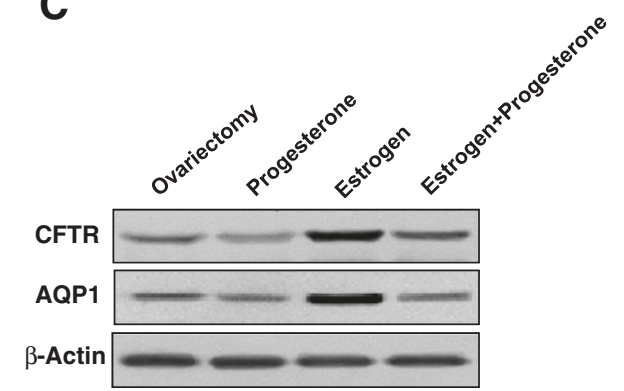

Figure 2 Estrogen upregulates CFTR and AQP1 expression in peritoneal epithelial cells and induces OHSS in rats. Serum estrogen concentrations in OHSS were significantly different from control rats at both estrous and diestrous stages of the estrous cycle $(\mathbf{A})$. ${ }^{* * *} P<0.001$ ( $n=6,6$, and 6 for diestrus, estrus, and OHSS, respectively). Comparison of increased WT of rat $30 \mathrm{~d}$ after ovariectomy (control) and after treatment with progesterone or estrogen for $5 d\left(n=5 /\right.$ group), showing significantly increased WT by estrogen (B). ${ }^{* * *} P<0.001$ (compared with the control). Western blot results showed downregulation of CFTR and AQP1 expression by progesterone and upregulation expression by estrogen in peritoneal epithelial cells of ovariectomized rats. The Pixel Value for CFTR: ovariectomy 260546, Progesterone 202730, Estrogen 885290, Estrogen+Progesterone 363422; The Pixel Value for AQP1: ovariectomy 186484, Progesterone 101092, Estrogen 877020, Estrogen +Progesterone 160054; The Pixel Value for $\beta$-Actin: ovariectomy 708508, Progesterone 707448, Estrogen 707982, Estrogen+Progesterone 709654 (C). 
but not progesterone, is responsible for the up regulation of CFTR and AQP1 leading to OHSS.

\section{Effect of OHSS on peritoneal epithelial CFTR channel activity in rats}

We compared CFTR activity between freshly isolated peritoneal epithelial cells of normal and OHSS rats by Isc measurement. In the presence of an epithelial sodium channel blocker, amiloride (to exclude a possible contribution of $\mathrm{Na}^{+}$absorption to the current observed), the forskolin-induced Isc from the peritoneal epithelia of OHSS rats (PMSG plus hCG) was substantially increased compared with that in untreated controls at the diestrous or estrous stages of the sexual cycle (Figure 3). The cAMP-dependent Isc could be blocked by a CFTR specific inhibitor-172 (Calbiochem, La Jolla, CA USA; Figure 3 A-D), confirming that the observed cAMPactivated Isc reflects CFTR channel activity. The results showed that CFTR channel activity was enhanced in OHSS.

\section{Effect of estrogen and progesterone on the peritoneal epithelial CFTR channel activity}

The effect of exogenously administrated estrogen and progesterone for $5 \mathrm{~d}$ on peritoneum CFTR channel activity was examined. Isc measurements showed that forskolin-induced or CFTR-mediated Isc in estrogentreated peritoneum (Figure $4 \mathrm{~B}$ and D) was significantly higher than that in progesterone-treated ones (Figure 4 $\mathrm{A}$ and $\mathrm{D})$. Besides, progesterone suppressed estrogen induced CFTR-mediated Isc in estrogen-treated peritoneum (Figure 4 C and D).

\section{Progesterone downregulated CFTR and AQP1 expression and suppressed OHSS rats}

Progesterone treatment $(25 \mathrm{mg} / \mathrm{rat} \cdot \mathrm{d})$ of PMSG/hCGinduced OHSS rats significantly reduced WT increase, ascites volume and pleural effusion volume (Figure 5 AC). Progesterone treatment of OHSS rats also significantly reduced CFTR and AQP1 protein expression (Figure $5 \mathrm{D}$ ). Besides, a selective progesterone antagonist, mifepristone irrigation significantly reduced the reverse of progesterone to OHSS symptoms of rats as well as CFTR and AQP1 expression reduction in peritoneal epithelial cells (Figure $5 \mathrm{~A}-\mathrm{D}$ ). It may be the reason why progesterone was found to significantly lower the incidence of OHSS in IVF cycles in a few clinical situations for luteal support during assisted reproductive technology (ART) [28-30].

\section{Coaction of the peritoneal epithelial CFTR and AQP1 channel activity in the development of OHSS}

We found that AQP1 inhibitor $\left(\mathrm{HgCl}_{2}\right)$ could suppress the CFTR activity in the freshly isolated peritoneal epithelial cells of OHSS rats by the short circuit current (Isc) measurement. For the OHSS rats (PMSG plus hCG), in the presence of an AQP1 inhibitor, $\mathrm{HgCl}_{2}$ (to block water exocrine), the forskolin-induced Isc from the peritoneal epithelial cells of OHSS rats (PMSG plus hCG) was substantially decreased compared with that in untreated controls of the OHSS rats (Figure 6 A-C). Furthermore, treatment of noncycling PMSG-/hCG-induced OHSS rats (to exclude any cyclic influence) with $300 \mu \mathrm{l}$ CFTR antiserum (1:100 dilution, vol/vol, in buffer; Zymed Laboratories) or $400 \mu \mathrm{l}$ AQP1 antiserum (1:100 dilution, vol/vol, in buffer; Santa Cruz Biotechnology) by
A

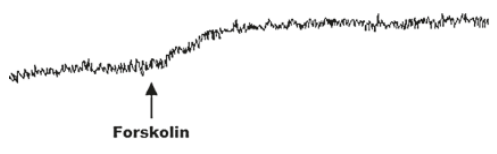

C

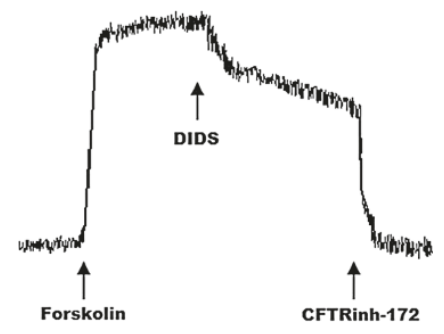

B

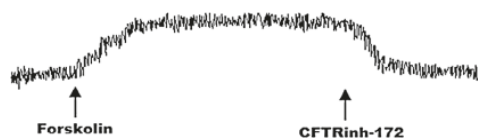

D

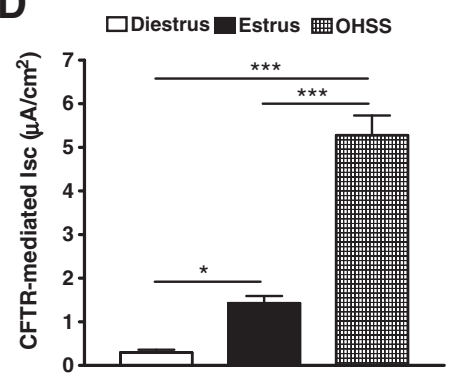

Figure 3 Effect of OHSS on peritoneal epithelial CFTR channel activity. CFTR-mediated peritoneum anion secretion measured by Isc in the presence of an epithelial $\mathrm{Na}^{+}$channel blocker, amiloride $(10 \mu \mathrm{M})$, showed an elevated forskolin $(10 \mu \mathrm{M})$ response in PMSG-/hCG-induced OHSS peritoneal epithelial cells $(\mathbf{C})$ compared with the controls at diestrous $(\mathbf{A})$ and estrous (B) stages of the sexual cycle (D). ${ }^{*} P<0.05,{ }^{* * *} P<0.001$ ( $n=4 /$ group, compared with the diestrus). 


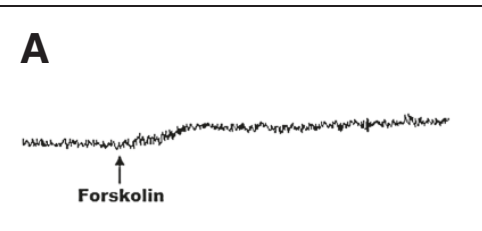

B

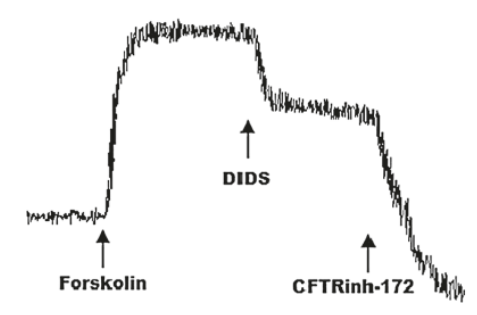

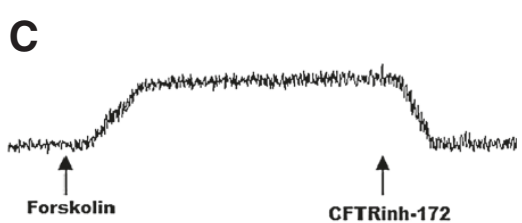

D

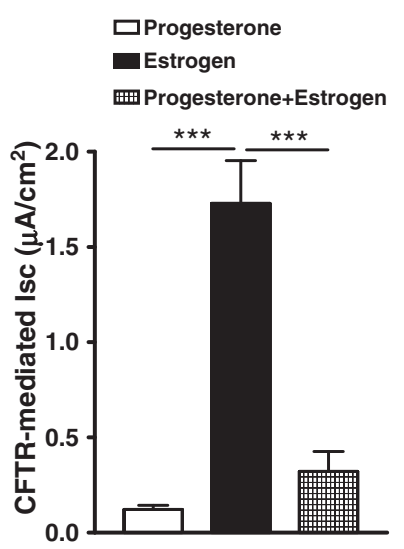

Figure 4 Effect of estrogen and progesterone on the peritoneal epithelial CFTR channel activity. Progesterone treatment ( $25 \mathrm{mg} / \mathrm{d} \cdot \mathrm{rat})$ of female rats for $5 \mathrm{~d}$ did not induce CFTR-mediated Isc in the peritoneal epithelial cells (A), but estrogen treatment (10 $\mu \mathrm{g} / \mathrm{kg}$ body weight.d) did (B). Besides, progesterone treatment suppressed estrogen induced CFTR-mediated IsC (C). Statistical analyses of forskolin-activated CFTR-mediated Isc response showed significant CFTR activity in the peritoneum of estrogen-treated rats $(\mathbf{C}) .{ }^{* * *} P<0.001(n=12)$.

IP injection to suppress the function of CFTR or AQP1 resulted in the elimination of body WT increase, ascites volumes and pleural effusion volumes (Figure 6 D-F).

\section{Discussion}

In addition to being a chloride channel, CFTR, may function as a regulator of other channels, including aquaporin water channels (channels that allow rapid flow of water across epithelial membranes) and epithelial sodium channels. Enhancement of aquaporin channel activity [31] and suppression of epithelial sodium channel-mediated absorptive activity [32] that results in net increases in electrolyte and fluid secretion may also contribute to the pathogenesis of OHSS.

To test this hypothesis, we developed the OHSS rat model by using several combinations of gonadotropins as previously described [33]. There was a marked increase in fluid accumulation in the peritoneal cavity and pleural cavity (Figure $1 \mathrm{~B}$ and C). Therefore, the increased body WT in the hyperstimulated rats was significantly different from those in the controls (Figure 1 A). Enhanced CFTR and AQP1 expression in body cavity peritoneal epithelial cells was observed in the OHSS rats (Figure 1 D). Thus, up-regulated CFTR and AQP1 expression in peritoneal epithelial cells is correlated with the fluid accumulation observed in the body cavity of OHSS animals. It has been well documented that OHSS patients have high plasma estrogen levels [26]. It is possible that hyper stimulation of ovaries leads to high production and release of estrogen, which is responsible for the upregulation of CFTR and AQP1 observed. This may explain why OHSS may not occur or may become severe despite high estrogen levels in some individuals who could have CFTR mutations and defective CFTR function.

Estrogen levels had been monitored in the induced OHSS rats as well as in the controls at both estrous and diestrous stages of the estrous cycle. The results showed that estrogen levels increased over 6-fold in the OHSS rats compared with that in the controls (Figure $2 \mathrm{~A}$ ), which is consistent with other OHSS animal models [33]. The relationship between elevation of estrogen alone and OHSS had been investigated by developing of OHSS symptoms in ovariectomized rats. It confirmed that hyper stimulation did not produce OHSS symptoms in these ovariectomized rats unless treated with estrogen, but not progesterone, for 5 days (Figure 2 B). Furthermore, progesterone suppressed estrogen induced OHSS symptom as well as CFTR and AQP1 expression upregulation (Figure $2 \mathrm{~B}$ and $\mathrm{C}$ ). Exogenous estrogen induced WT increase as well as CFTR and AQP1 expression upregulation in ovariectomized rats, similar to those in the OHSS rat model without ovariectomy (Figure $2 \mathrm{~B}$ and $\mathrm{C}$ ). It should be noted that although estrogen drastically increased CFTR and AQP1 expression, progesterone completely suppressed both of them in 
A
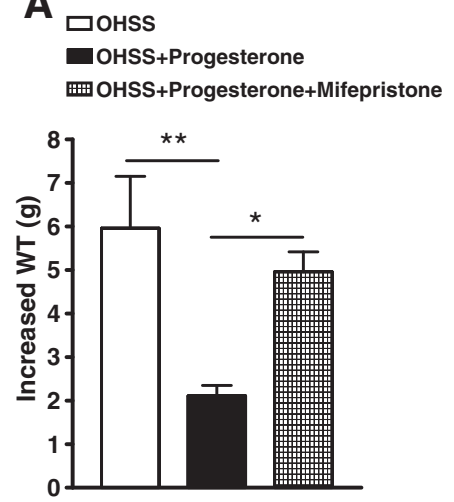

C 口онss OHSS+Progesterone 曲 OHSS+Progesterone+Mifepristone

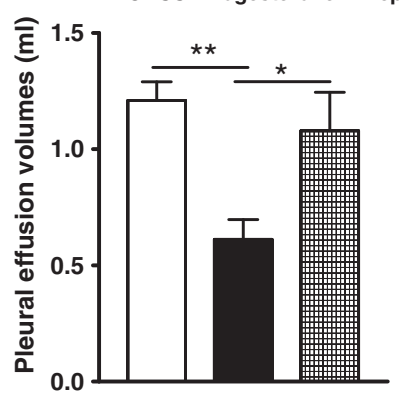

B

口OHSS

COHSS+Progesterone

回OHSS+Progesterone+Mifepristone

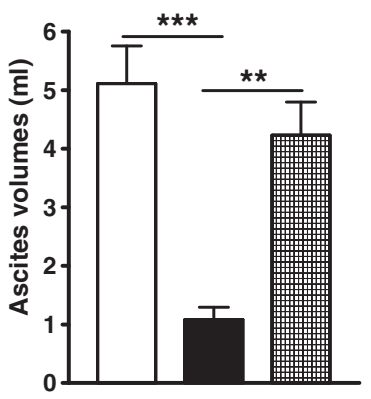

D

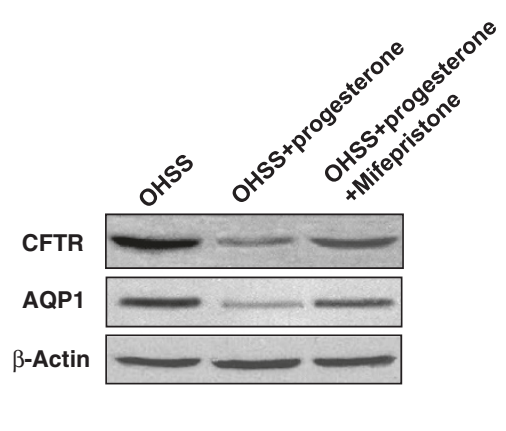

Figure 5 Effect of progesterone on the expression of CFTR and AQP1 expression in peritoneal epithelial cells and OHSS symptoms in rats. Statistical comparison of WT increase (A), ascites volume (B), and pleural effusion volume (C) in PMSG-/hCG-induced OHSS rats and OHSS rats [treated with progesterone, $25 \mathrm{mg} / \mathrm{d} \cdot \mathrm{rat}$; or treated with both progesterone $(25 \mathrm{mg} / \mathrm{d} \cdot \mathrm{rat}$ ) and mifepristone (diluted in corn oil, $10 \mathrm{mg} / \mathrm{d} \cdot \mathrm{rat}$, irrigation daily), $\mathrm{n}=5$ /group]. ${ }^{*} P<0.05,{ }^{* *} P<0.01,{ }^{* *} P<0.001$. Western blot results showed that expression of CFTR and AQP1 proteins in peritoneal epithelial cells were suppressed by progesterone in the OHSS rats. The Pixel Value for CFTR: OHSS 1005806, OHSS+progesterone 223260, OHSS+progesterone+mifepristone 394564; The Pixel Value for AQP1: OHSS 424325, OHSS+progesterone 183016, OHSS+progesterone +mifepristone 248076; The Pixel Value for $\beta$-Actin: OHSS 821654, OHSS+progesterone 824855, OHSS+progesterone+mifepristone 823090 (D).

ovariectomized rats. This is consistent with previous results obtained in ovary-intact animals showing upregulation and downregulation of CFTR by estrogen and progesterone, respectively [17]. OHSS symptoms and CFTR upregulation in peritoneal epithelial cells were also observed in ovary-intact rats treated with high levels of estrogen, but not progesterone (Figure $2 \mathrm{~B}$ and $\mathrm{C}$ ). The serum level of exogenously administrated estrogen was similar to the estrogen levels in OHSS rats (Figure 2 $B$ and $C$ ). These results suggest that the ovarian sex hormone, estrogen, but not progesterone, is responsible for the upregulation of CFTR and AQP1 leading to OHSS. These results support the clinical observation that OHSS rarely develops in individuals undergoing ovarian hyper stimulation in the absence of high estrogen levels [26].

To confirm that up regulation of CFTR indeed leads to enhancement of its channel activity, from where excessive epithelial fluid secretion derives, we compared functional CFTR activity between freshly isolated peritoneal epithelial cells of normal and OHSS rats by the
Isc measurement [34]. To exclude a possible contribution of $\mathrm{Na}^{+}$absorption to the current, we used amiloride, an epithelial sodium channel blocker. The results revealed that the forskolin-induced Isc from the peritoneal epithelial cells of OHSS rats was substantially increased compared with that of untreated controls at the diestrous or estrous stage of the sexual cycle (CFTR is known to be minimally and maximally expressed, respectively). The cAMP-dependent Isc could be blocked by a specific CFTR inhibitor-172 (Figure 3 A-D), which has been demonstrated to inhibit CFTR-mediated, cholera toxin-induced fluid secretion [35]. It confirmed that the observed cAMP-activated Isc reflects CFTR channel activity. The effect of exogenously administrated estrogen and progesterone for $5 \mathrm{~d}$ on peritoneal CFTR channel activity was also examined. Isc measurements showed that forskolin-induced or CFTR-mediated Isc in estrogen-treated peritoneum was significantly higher than that of progesterone-treated (Figure $4 \mathrm{~A}$ and $\mathrm{B}$ ). Progesterone suppressed estrogen induced CFTR- 


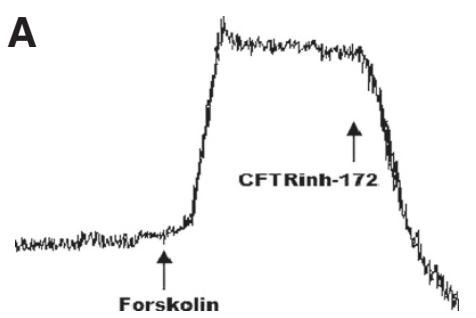

B

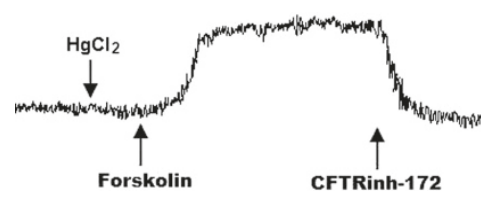

D $\square$ OHSS 西 OHSS+AQP1 antiserum

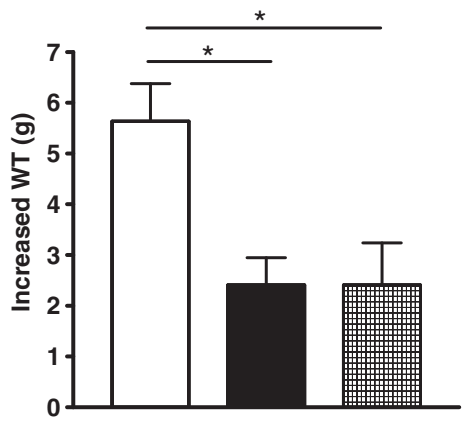

F $\quad$ OHSS O OHSS+AQP1 antiserum

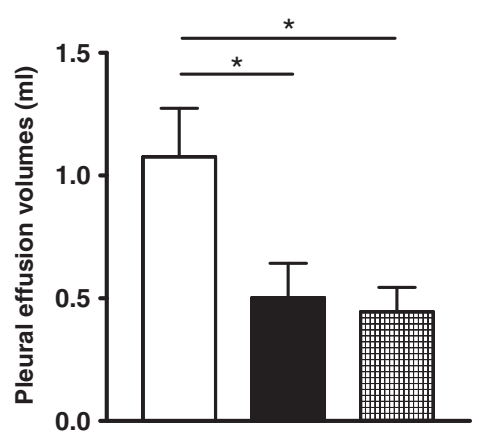

C

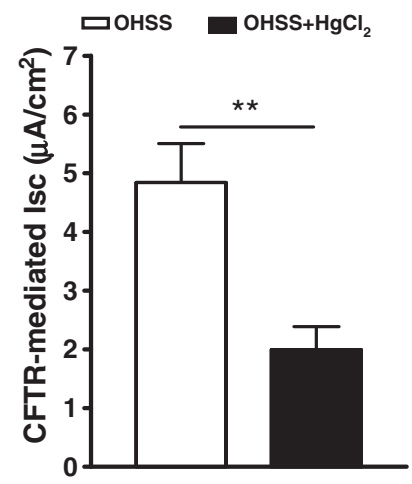

E 口 OHSS

- OHSS+CFTR antiserum 回 OHSS+AQP1 antiserum

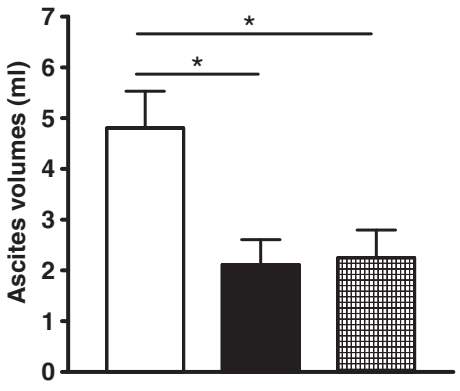

Figure 6 Coactions of CFTR and AQP1 in the excessive fluid transport in OHSS. The $\mathrm{HgCl}_{2}$ pre-treatment (30 $\mu$ M) suppressed CFTRmediated Isc in the peritoneal epithelial cells (A, B and $\mathbf{C})$, showing Coaction of CFTR and AQP1 activity in the peritoneal epithelial cells. ${ }^{* *} P<0.01$

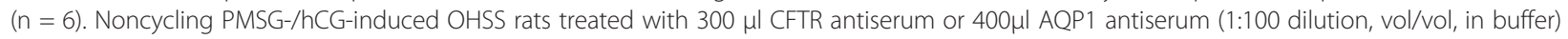
significantly reduced WT increase $(\mathbf{D})$, ascites volumes $(\mathbf{E})$ and pleural volumes $(\mathbf{F})$ compared with the heat-inactivated normal rat serum (NRS)-treated controls. ${ }^{*} P<0.05(n=6)$.

mediated Isc in peritoneum (Figure $4 \mathrm{C}$ ). Taken together, it appears that up regulation of CFTR and AQP1 due to elevated estrogen may be responsible for the excessive fluid transport and accumulation in OHSS. Therefore, it may be possible to alleviate OHSS symptoms through suppressing CFTR or AQP1 expression or through suppressing CFTR or AQP1 function. To validate this hypothesis, we first used progesterone, as it was shown to suppress CFTR expression in previous studies [17] and in the present study as well. Indeed, progesterone treatment of OHSS rats also eliminated peritoneal and pleural fluid accumulation and significantly reduced CFTR and AQP1 protein expression (Figure 5 A-D). The progesterone selective progesterone antagonist, 
mifepristone, was found to suppress the action of progesterone (Figure $5 \mathrm{~A}-\mathrm{D}$ ). Interestingly, progesterone has been used in a few clinical situations for luteal support during ART, in which it was found to significantly lower the incidence of OHSS in IVF cycles. However no explanation for such an effect was published [2830]. In summary, progesterone may suppress the expression of CFTR and AQP1 to alleviate the symptom of OHSS.

To confirm the coactions of CFTR and AQP1 in the pathogenesis of OHSS, the cAMP-dependent Isc was used to measure CFTR channel activity affected by AQP1. The results showed that the cAMP-dependent Isc could also be reduced by an AQP1 inhibitor, $\mathrm{HgCl}_{2}$ (Figure $6 \mathrm{~A}-\mathrm{C}$ ), which has been demonstrated to inhibit AQP1-mediated water transport [36]. This confirmed that AQP1 can affect CFTR channel activity. Furthermore, treatment of PMSG-/hCG-induced OHSS rats injected with $300 \mu \mathrm{l}$ CFTR antiserum resulted in the elimination of the body WT increase about $3.23 \mathrm{~g}$, the peritoneal fluid accumulation about $2.69 \mathrm{ml}$ and the pleural fluid accumulation about $0.58 \mathrm{ml}$. Injection of $300 \mu \mathrm{l}$ AQP1 antiserum resulted in the elimination of the body WT increase about $3.23 \mathrm{~g}$, the peritoneal fluid accumulation about $2.56 \mathrm{ml}$ and the pleural fluid accumulation about $0.64 \mathrm{ml}$ (Figure $6 \mathrm{D}-\mathrm{F}$ ). The ability to alleviate OHSS symptoms through interfering with CFTR or AQP1 function confirmed a critical role of the coactions of CFTR and AQP1 in OHSS pathogenesis. It also suggested that these treatments may be a preventive measure for OHSS during ART.

In this study, we demonstrated that up regulation of both CFTR and AQP1 by either excessive estrogen or ovarian hyper stimulation leads to the development of OHSS symptoms in rodents. Considering the observed high levels of estrogen, exceeding normal physiological levels, in patients undergoing ovarian hyper stimulation $[25,26]$ and regulation of CFTR and AQP1 expression by ovarian hormones in humans [14], the present findings in OHSS rodent models are of strong clinical relevance, suggesting the coactions of CFTR and AQP1 in the pathogenesis of OHSS. OHSS appears to be the result of abnormally up regulating CFTR as well as AQP1. This leads to excessive fluid shift and accumulation in the peritoneal cavity and pleural cavity, which can be life threatening. It is because that fluid transport throughout the body due to water effusion following ion transportation.

\section{Conclusions}

Taken together, this study confirms the coactions of CFTR and AQP1 in regulating body electrolyte and fluid secretion. An abnormality in either expression or function, could result in lethal conditions, as seen in OHSS.

\section{Methods}

\section{Animals}

Prior approval of the experimental protocols was obtained from the local ethics committee at Zhengzhou University for use of animals in experiments. The Sprague-Dawley (S-D) rats were used in this study. Animals were maintained in the Laboratory Animal Service Center of Zhengzhou University before experiments and were kept in a temperature-controlled room with a 12L:12D cycle and with food and water ad libitum. All animal experiments were conducted in accordance with the university Laboratory Animals Service Center's guidelines on animal experimentation.

\section{Ovariectomy}

The animals were 13 weeks of age (when rats reach sexual maturation), weighing about 250-300 g. General anesthesia was administered using intraperitoneal (IP) injection of a mixture of $2 \%$ xylazine hydrochloride (10 $\mathrm{mg} / \mathrm{kg}$ ) (Alfasan, Woerden, Holland) and ketamine hydrochloride $(80 \mathrm{mg} / \mathrm{kg}$ ) (Alfasan). With the use of sharp dissecting scissors, both oviducts were exposed, one at a time, after careful dissection and bilateral oviductal ligation were performed, and ovaries were removed. The uterine horns were gently put back into the peritoneal cavity. The muscle layers were approximated with absorbable suture, skin incisions were closed using surgical clips, and adequate postoperative care was given. Rats were kept for at least $30 \mathrm{~d}$ to recover and adjust before additional experiments.

\section{OHSS induction}

Female S-D rats at 13 weeks old were divided into four groups ( $n=5 /$ group). All rats were weighed before the experiment. To prepare the OHSS model, the rats are stimulated with human menopausal gonadotropin (HMG, Serono Laboratories, Aubonne, Switzerland), follicle stimulating hormone (FSH, Organon NV, Oss, The Netherlands), or pregnant mares' serum gonadotrophin (PMSG, Sigma-Aldrich Corp., St. Louis, MO, USA) in conjunction with hCG (Organon NV) as previously reported [33]. Gonadotropins were administered by IP injections. PMSG (300 IU/kg body weight), HMG (75 $\mathrm{IU} /$ rat.d), and FSH (75 IU/rat.d) were injected for $4 \mathrm{~d}$ consecutively; on the fifth day, rats received 300 IU hCG/rat. Control animals received phosphate buffered saline (PBS) for the same period. Serum concentrations of estradiol is also increased.

\section{Western blot analysis}

Tissue samples, peritoenal biopsy, were lysed with modified RIPA buffer (50 mM Tris-HCl, pH 7.4; $150 \mathrm{mM}$ $\mathrm{NaCl}, 1 \% \mathrm{NP} 40,0.25 \%$ Na-deoxycholate, $1 \mathrm{mM}$ EDTA) supplemented with $1 \mathrm{mM}$ PMSF to inhibit proteolytic 
enzyme. The whole cell lysates containing equal amounts of protein $(20-30 \mu \mathrm{g})$ were separated on 10$12 \%$ sodium dodecyl sulphate polyacrylamide gel electrophoresis (SDS-PAGE) and transferred to HybondECL nitrocellulose membranes (Amersham, USA). Membrane was blocked using $4 \%$ skimmed milk and then incubated with anti-CFTR antibody (Zymed Laboratories, San Fransisco, CA, USA), $\beta$-Actin (Santa Cruz Biotechnology, Santa Cruz, CA, USA) and antiAQP1 antibody (Santa Cruz Biotechnology). The blots were incubated with HRP-conjugated goat anti-rabbit secondary antibodies (Santa Cruz Biotechnology) and were visualized by Enhanced Chemiluminescence (ECL) (Amersham Biosciences, USA) following light sensitive films (Fuji, Japan) development. Integrated density values were calculated using Alpha Imager 3400 (Alpha InnoTech, USA). The actual values of the western blot bands were quantified using MetaMorph image analysis software (Universal Imaging Corporation, USA). And the quantities of the protein were valued by pixel value. All experiments were repeated at least three times and the representative results were presented.

\section{Short-circuit current (Isc) measurement}

Isc measurement has previously been described [34]. In brief, freshly removed parietal peritoneum from which the muscular layers had been removed were clamped vertically between two halves of the Ussing chamber. The peritoneal epithelial cells were bathed on both sides with Krebs-Henseleit solution that was maintained at 37 C. The Krebs-Henseleit solution had the following composition: $117 \mathrm{mM} \mathrm{NaCl}, 4.7 \mathrm{mM} \mathrm{KCl}, 2.5 \mathrm{mM} \mathrm{CaCl} 2$, $1.2 \mathrm{mM} \mathrm{MgCl} 2,24.8 \mathrm{mM} \mathrm{NaHCO} 3,1.2 \mathrm{mM} \mathrm{KH} 2 \mathrm{PO} 4$, and $11.1 \mathrm{mM}$ glucose and it was bubbled with $95 \% \mathrm{O} 2$ and $5 \% \mathrm{CO} 2$ to maintain the $\mathrm{pH}$ at 7.4. Drugs were added directly to the apical or basolateral side of the epithelial cells. The transepithelial potential differences exhibited by the epithelial cells were measured by the $\mathrm{Ag} / \mathrm{AgCl}$ electrodes (World Precision Instruments, Sarasota, FL, USA) connected to a preamplifier, which was connected to a voltage clamp amplifier (DVC 1000, World Precision Instruments). The change in Isc was defined as the maximal rise in Isc after agonist stimulation and was normalized as current change per unit area of the peritoneal epithelial cells (microamperes per square centimeter).

\section{Hormonal measurement}

Serum estrogen concentrations were measured using an electro-chemiluminescence immunoassay by means of the Elecsys Estradiol 11 kit with the automated Elecsys Immunoanalyzer (Roche, Mannheim, Germany).

\section{Statistical analysis}

Data are presented as the means \pm SEM. Multiple comparisons and differences between groups were analyzed using the one-way ANOVA test, and paired data were analyzed by unpaired $t$ test. $P<0.05$ (two-tailed) was considered statistically significant. Analyses were carried out with PRISM (GraphPad, Inc., San Diego, CA, USA).

\section{Competing interests}

The authors declare that they have no competing interests related to the manuscript.

\section{Authors' contributions}

L.L. conceived and designed the experiments, and revision of the paper critically for important intellectual content and final approval of the version to be published. P.Y.J. performed the experiments. Y.C.L and P.Y.J revised the paper. P.Y.J. and Q.F.H. analyzed the data. P.Y.J., Q.F.H. and L.L. drafted the article. All authors read and approved the final manuscript.

\section{Acknowledgements}

This work was supported by the Natural Science Foundation of Henan Province (Grant No.2009285101263855)

\section{Author details}

'Department of Cardiovascular Medicine, The First Affiliated Hospital of Zhengzhou University, Zhengzhou, Henan 450052, China. ²Department of Reproductive Technology, Henan Provincial institute for Polulation and Family Planning Research, Zhengzhou, Henan 450002, China. ${ }^{3}$ Department of Cardiovascular Medicine, The People's Hospital of Anyang, Anyang, Henan 455000, China

Received: 25 December 2011 Accepted: 22 August 2012

Published: 28 August 2012

\section{References}

1. Navot D, Bergh PA, Laufer N: Ovarian hyper stimulation syndrome in novel reproductive technologies: prevention and treatment. Fertil Steril 1992, 58:249-261.

2. Balasch J, Fabregues F, Arroyo V: Peripheral arterial vasodilation hypothesis: a new insight into the pathogenesis of ovarian hyper stimulation syndrome. Hum Reprod 1998, 13:271-273.

3. Navot D, Bergh PA, Laufer N: The ovarian hyper stimulation syndrome. In Reproductive endocrinology, surgery and technology. Edited by Adashi EY, Rock JA, Rosenwaks Z. Philadelphia: Lippincott-Raven; 1995:2215-2232.

4. Pellicer A, Albert C, Mercader A, Bonilla-Musoles F: Remohi' J, Simo'n C: The pathogenesis of ovarian hyper stimulation syndrome: in vivo studies investigating the role of interleukin (IL)-1 $\beta$, IL- 6 and vascular endothelial growth factor (VEFG). Fertil Steril 1999, 71:482-489.

5. Go'mez R, Simo'n C, Remohi' J, Pellicer A: Vascular endothelial growth factor receptor-2 activation induces vascular permeability in hyperstimulated rats, and this effect is prevented by receptor blockade. Endocrinology 2002, 143:4339-4348.

6. Go'mez R, Simo'n C, Remohı' J, Pellicer A: Administration of moderate and high doses of gonadotropins to female rats increases ovarian vascular endothelial growth factor (VEGF) and VEGF receptor-2 expression that is associated to vascular hyperpermeability. Biol Reprod 2003, 68:2164-2171.

7. Garci'A-Velasco JA, Zun iga A, Pacheco A, Go'mez R, Simo'n C, Remohi' J, Pellicer A: Coasting acts through downregulation of VEGF gene expression and protein secretion. Hum Reprod 2004, 19:1530-1538.

8. Verkman AS: Aquaporins in endothelia. Kidney Int 2006, 69:1120-1123.

9. Gabriel SE, Brigman KN, Koller BH, Boucher RC, Stutts MJ: Cystic fibrosis heterozygote resistance to cholera toxin in the cystic fibrosis mouse model. Science 1994, 266:107-109.

10. Kerem B, Rommens JM, Buchanan JA, Markiewicz D, Cox TK, Chakravarti A, Buchwald M, Tsui LC: Identification of the cystic fibrosis gene: genetic analysis. Science 1989, 245:1073-1080

11. Quinton PM: Physiological basis of cystic fibrosis: a historical perspective. Physiol Rev 1999, 79:S3-S22. 
12. Ajonuma LC, Tsang LL, Zhang GH, Wong HY, Lau MC, Ho LS, Rowlands DK, Zhou CX, Ng CP, Chen J, Xu PH, Zhu JX, Chung YW, Chan HC: Estrogeninduced abnormally high cystic fibrosis transmembrane conductance regulator expression results in ovarian hyper stimulation syndrome. $\mathrm{Mol}$ Endocrinol 2005, 19:3038-3044.

13. Trezise AE, Buchwald M: In vivo cell-specific expression of the cystic fibrosis transmembrane conductance regulator. Nature 1991, 353:434-437.

14. Tizzano EF, Silver MM, Chitayat D, Benichou JC, Buchwald M: Differential cellular expression of cystic fibrosis transmembrane regulator in human reproductive tissues. Clues for the infertility in patients with cystic fibrosis. Am J Pathol 1994, 44:906-914.

15. Rochwerger $L$, Buchwald M: Stimulation of the cystic fibrosis transmembrane conductance regulator expression by estrogen in vivo. Endocrinology 1993, 133:921-930.

16. Rochwerger L, Dho S, Parker L, Foskett JK, Buchwald M: Estrogendependent expression of the cystic fibrosis transmembrane conductance regulator gene in a novel uterine epithelial cell line. J Cell Sci 1994, 107:2439-2448.

17. Mularoni A, Beck L, Sadir R, Adessi GL, Nicollier M: Down-regulation by progesterone of CFTR expression in endometrial epithelial cells: a study by competitive RT-PCR. Biochem Biophys Res Commun 1995, 217: 1105-1111.

18. Chan LN, Chung YW, Leung PS, Liu CQ, Chan HC: Activation of an adenosine 3,5-cyclic monophosphatedependent $\mathrm{Cl}^{-}$conductance in response to neurohormonal stimuli in mouse endometrial epithelial cells: the role of cystic fibrosis transmembrane conductance regulator. Biol Reprod 1999, 60:374-380.

19. Chan LN, Tsang LL, Rowlands DK, Rochelle LG, Boucher RC, Liu CQ, Chan $\mathrm{HC}$ : Distribution of epithelial sodium channels $(\mathrm{ENaC})$ subunits and cystic fibrosis transmembrane conductance regulator (CFTR) in murine reproductive tract. J Membr Biol 2002, 185:165-176.

20. Nielsen S, Smith BL, Christensen El, Agre P: Distribution of the aquaporin CHIP in secretory and resorptive epithelia and capillary endothelia. Proc Natl Acad Sci 1993, 90:7275-7279.

21. Hasegawa H, Lian SC, Finkbeiner WE, Verkman AS: Extrarenal tissue distribution of CHIP28 water channels by in situ hybridization and antibody staining. Am J Physiol 1994, 266:C893-C903.

22. Yang BX, Folkesson HG, Yang J, Matthay MA, Ma TH, Verkman AS: Reduced osmotic water permeability of the peritoneal barrier in aquaporin-1 knockout mice. Am J Physiol Cell Physiol 1999, 276:76-81.

23. Richard C, Gao J, Brown N, Reese J: Aquaporin water channel genes are differentially expressed and regulated by ovarian steroids during the periimplantation period in the mouse. Endocrinology 2003, 144:1533-1541.

24. Lindsay LA, Murphy CR: Redistribution of aquaporins 1 and 5 in the rat uterus is dependent on progesterone: a study with light and electron microscopy. Reproduction 2006, 131:369-378.

25. Manau D, Balasch J, Arroyo V, Jimenez W, Fabregues F, Casamitjana R, Creus $M$, Vanrell JA: Circulatory dysfunction in asymptomatic in vitro fertilization patients: relationship with hyperestrogenemia and activity of endogenous vasodilators. J Clin Endocrinol Metab 1998, 83:1489-1493.

26. Raziel A, Friedler S, Schachter M, Strassburger D, Mordechai E, Ron-El R: Increased early pregnancy loss in IVF patients with severe ovarian hyper stimulation syndrome. Hum Reprod 2002, 17:107-110.

27. Delvigne A, Rozenberg S: Epidemiology and prevention of ovarian hyper stimulation syndrome (OHSS): a review. Hum Reprod Update 2002, 8:559-577.

28. Costabile L, Unfer V, Manna C, Gerli S, Rossetti D, Di Renzo GC: Use of intramuscular progesterone versus intravenous albumin for the prevention of ovarian hyper stimulation syndrome. Gynecol Obstet Invest 2000, 50:182-185.

29. Ma T, Thiagarajah JR, Yang H, Sonawane ND, Folli C, Galietta LJ, Verkman AS: Thiazolidinone CFTR inhibitor identified by high-throughput blocks cholera toxin-induced intestinal fluid secretion. J Clin Invest 2002, 110:1651-1658.

30. Penzias AS: Luteal phase support. Fertil Steril 2002, 77:318-323.

31. Schreiber R, Nitschke R, Greger R, Kunzelmann K: The cystic fibrosis transmembrane conductance regulator activates aquaporin 3 in airway epithelial cells. J Biol Chem 1999, 274:11811-11816.
32. Stutts MJ, Canessa CM, Olsen JC, Hamrick M, Cohn JA, Rossier BC, Boucher RC: CFTR as a CAMP-dependent regulator of sodium channels. Science 1995, 269:847-850.

33. Ohba T, Ujioka T, Ishikawa K, Tanaka N, Okamura H: Ovarian hyper stimulation syndrome-model rats; the manifestation and clinical implication. Mol Cell Endocrinol 2003, 202:47-52.

34. Ussing $\mathrm{HH}$, Zerahn $\mathrm{K}$ : Active transport of sodium as the source of electric current in the short-circuited isolated frog skin. Acta Physiol Scand 1951, 23:110-127.

35. Schwarzler P, Abendstein BJ, Klingler A, Kreuzer E, Rjosk HK: Prevention of severe ovarian hyper stimulation syndrome (OHSS) in IVF patients by steroidal ovarian suppression-a prospective randomized study. Human Fertil 2003, 6:125-129.

36. Preston GM, Jung JS, Guggino WB, Agre P: The mercury-sensitive residue at cysteine 189 in the CHIP28 water channel. J Biol Chem 1993, 268:17-20.

doi:10.1186/1471-2121-13-23

Cite this article as: Jin et al:: Co action of CFTR and AQP1 increases permeability of peritoneal epithelial cells on estrogen-induced ovarian hyper stimulation syndrome. BMC Cell Biology 2012 13:23.

\section{Submit your next manuscript to BioMed Central and take full advantage of:}

- Convenient online submission

- Thorough peer review

- No space constraints or color figure charges

- Immediate publication on acceptance

- Inclusion in PubMed, CAS, Scopus and Google Scholar

- Research which is freely available for redistribution 